\title{
Lower bound for the blow-up time for some nonlinear parabolic equations
}

\section{Wenhui Chen and Yan Liu*}

\section{"Correspondence:} liuyan99021324@tom.com Department of Applied Mathematics, Guangdong University of Finance, Guangzhou, 510521, P.R. China

\section{Springer}

\begin{abstract}
In this paper, we study the blow-up phenomenon for some nonlinear parabolic problems. Using the technique of differential inequalities, the lower bound for the blow-up time is determined if a blow-up does really occur. Our result is obtained in a bounded domain $\Omega \in \mathbb{R}^{N}$ for any $N \geq 3$.
\end{abstract}

Keywords: lower bound; blow-up time; nonlinear parabolic problems

\section{Introduction}

Payne et al. [1] studied the blow-up phenomenon for solutions to the following family of mixed problems:

$$
\begin{aligned}
& \frac{\partial u}{\partial t}=\left(\rho\left(|\nabla u|^{2}\right) u_{, i}\right)_{, i}+f(u) \quad \text { in } \Omega \times\left(0, t^{*}\right), \\
& u(x, 0)=g(x) \geq 0 \quad \text { in } \Omega, \\
& u(x, t)=0 \quad \text { in } \partial \Omega \times\left(0, t^{*}\right) .
\end{aligned}
$$

They obtained a lower bound for the blow-up time $t^{*}$ if the blow-up does really occur together with a criterion for getting a blow-up. Moreover, they proposed conditions that ensure that a blow-up cannot occur. In this paper, we continue the work of Payne, Philippin, and Schaefer. In [1], they obtained the lower bound for the blow-up time of solutions in a bounded domain $\Omega \in \mathbb{R}^{N}$ for $N=3$. If one is interested in generalizations to the case $N>3$, then one important tool, which is important for proving the results obtained in [1], namely, the Sobolev inequality is no longer applicable. There are only a few papers dealing with a lower bound for the blow-up time when $N>3$ (see [2,3]). Our goal is to get a lower bound for the blow-up time of the solutions to (1.1)-(1.3) in $\Omega \in \mathbb{R}^{N}$ for any $N \geq 3$.

The study of finite-time blow-up of solutions to parabolic problems under a homogeneous Dirichlet boundary condition and Neumann condition has earned great attention (see [4-10]). Recently, some papers began to consider the blow-up phenomena of these problems under the Robin boundary conditions (see [11-14]). Many methods have been used to study equations (1.1)-(1.3) (see [15-17]).

In this paper, $\Omega$ is a bounded star-shaped domain in $\mathbb{R}^{N}(N \geq 3)$ with smooth boundary $\partial \Omega$. The operator $\nabla$ is the gradient operator, and $t^{*}$ is the possible blow-up time. Furthermore, $i$ stands for the partial differentiation with respect to $x_{i}, i=1,2,3, \ldots, N$. The

(c) 2016 Chen and Liu. This article is distributed under the terms of the Creative Commons Attribution 4.0 International License (http://creativecommons.org/licenses/by/4.0/), which permits unrestricted use, distribution, and reproduction in any medium, provided you give appropriate credit to the original author(s) and the source, provide a link to the Creative Commons license, and indicate if changes were made. 
repeated index indicates Einstein's summation convention over the indices. We assume that $\rho$ is a positive $C^{1}$ function that satisfies

$$
\rho(s)+2 s \rho^{\prime}(s)>0, \quad s>0,
$$

so that $\left(\rho u_{i}\right), i$ is an elliptic operator. We also assume that $\rho$ and $f$ satisfy the conditions

$$
0<f(s) \leq a_{1}+a_{2} s^{p}, \quad s>0,
$$

and

$$
\rho(s) \geq b_{1}+b_{2} s^{q}, \quad s>0,
$$

where $p>1$ and $0<2 q<p-1$, and $a_{1}, a_{2}, b_{1}, b_{2}$ are positive constants. Using the maximum principle, we can get that $u$ is nonnegative in $x$ and $t \in\left[0, t^{*}\right)$.

In the further discussions, we will use the following Hölder inequality:

$$
\int_{\Omega} w^{x_{1}+x_{2}} d x \leq\left(\int_{\Omega} w^{\frac{x_{1}}{\alpha}} d x\right)^{\alpha}\left(\int_{\Omega} w^{\frac{x_{2}}{1-\alpha}} d x\right)^{1-\alpha},
$$

where $0<\alpha<1$, and $x_{1}, x_{2}$ are positive constants.

\section{Lower bound for the blow-up time}

In this section, we define the auxiliary function $\varphi=\varphi(t)$ as follows (see [1]):

$$
\varphi(t)=\int_{\Omega} u^{2(n-1)(q+1)+2} d x=\int_{\Omega} u^{\sigma} d x \quad \text { with } \sigma=2(n-1)(q+1)+2 .
$$

We establish the following theorem.

Theorem 1 Assume that $u=u(x, t)$ is the classical nonnegative solution of the mixed problem (1.1)-(1.3) in a bounded domain $\Omega \in \mathbb{R}^{N}(N \geq 3)$. Then the quantity $\varphi(t)$ defined in (2.1) satisfies the differential inequality

$$
\varphi^{\prime}(t) \leq \sigma a_{1}|\Omega|^{\frac{1}{\sigma}}[\phi(t)]^{\frac{\sigma-1}{\sigma}}+k_{1}[\phi(t)]^{\frac{(N-2) \alpha}{N \alpha-2}}+k_{2}[\phi(t)]^{\frac{(N-2) \alpha^{\prime}}{N \alpha^{\prime}-2}},
$$

which yields that the blow-up time $t^{*}$ is bounded from below. We have

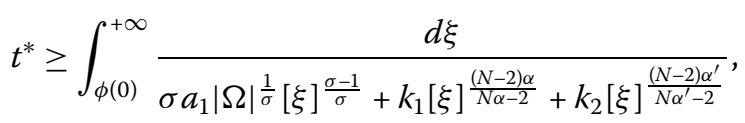

where $|\Omega|$ is the volume of the domain $\Omega$, and $k_{1}, k_{2}$ are positive constants that will be defined later.

Proof First, we compute

$$
\begin{aligned}
\varphi^{\prime}(t) & =\sigma \int_{\Omega} u^{\sigma-1}\left[\left(\rho\left(|\nabla u|^{2}\right) u_{, i}\right)_{, i}+f(u)\right] d x \\
& =-\sigma(\sigma-1) \int_{\Omega} u^{\sigma-2} \rho\left(|\nabla u|^{2}\right)|\nabla u|^{2} d x+\sigma \int_{\Omega} u^{\sigma-1} f(u) d x
\end{aligned}
$$


Chen and Diu Boundary Value Problems ( 2016) 2016:161

Page 3 of 6

$$
\begin{aligned}
\leq & -\sigma(\sigma-1) \int_{\Omega} u^{2(n-1)(q+1)}|\nabla u|^{2}\left(b_{1}+b_{2}|\nabla u|^{2 q}\right) d x \\
& +\sigma \int_{\Omega} u^{\sigma-1}\left(a_{1}+a_{2} u^{p}\right) d x .
\end{aligned}
$$

Using the equality

$$
\left|\nabla u^{n}\right|^{2(q+1)}=\left|n u^{n-1} \nabla u\right|^{2(q+1)}=n^{2(q+1)} u^{2(n-1)(q+1)}|\nabla u|^{2(q+1)}
$$

and the Hölder inequality, we get

$$
\varphi^{\prime}(t) \leq-\frac{\sigma(\sigma-1) b_{2}}{n^{2(q+1)}} \int_{\Omega}\left|\nabla u^{n}\right|^{2(q+1)} d x+\sigma a_{1}|\Omega|^{\frac{1}{\sigma}}[\phi(t)]^{\frac{\sigma-1}{\sigma}}+\sigma a_{2} \int_{\Omega} u^{\sigma+p-1} d x .
$$

If we set $v=u^{n}$, then we obtain

$$
\varphi^{\prime}(t) \leq-\frac{\sigma(\sigma-1) b_{2}}{n^{2(q+1)}} \int_{\Omega}|\nabla v|^{2(q+1)} d x+\sigma a_{1}|\Omega|^{\frac{1}{\sigma}}[\phi(t)]^{\frac{\sigma-1}{\sigma}}+\sigma a_{2} \int_{\Omega} v^{2(q+1)+\frac{\gamma}{n}} d x
$$

where $\gamma=p-1-2 q>0$. After application of the Hölder and Schwartz inequalities, it follows

$$
\begin{aligned}
\int_{\Omega}\left|\nabla v^{q+1}\right|^{2} d x & \leq(q+1)^{2}\left(\int_{\Omega}|\nabla v|^{2(q+1)} d x\right)^{\frac{1}{q+1}}\left(\int_{\Omega}|v|^{2(q+1)} d x\right)^{\frac{q}{q+1}} \\
& \leq(q+1) \int_{\Omega}|\nabla v|^{2(q+1)} d x+(q+1) q \int_{\Omega}|v|^{2(q+1)} d x
\end{aligned}
$$

Combining (2.6) and (2.7), we easily obtain

$$
\begin{aligned}
\varphi^{\prime}(t) \leq & -\frac{\sigma(\sigma-1) b_{2}}{n^{2(q+1)}(q+1)} \int_{\Omega}\left|\nabla v^{q+1}\right|^{2} d x+\frac{q \sigma(\sigma-1) b_{2}}{n^{2(q+1)}} \int_{\Omega} v^{2(q+1)} d x+\sigma a_{1}|\Omega|^{\frac{1}{\sigma}}[\phi(t)]^{\frac{\sigma-1}{\sigma}} \\
& +\sigma a_{2} \int_{\Omega} v^{2(q+1)+\frac{\gamma}{n}} d x .
\end{aligned}
$$

We choose $x_{1}, x_{2}$, and $\alpha$ such that

$$
x_{1}+x_{2}=2(q+1), \quad x_{1} \cdot \frac{1}{\alpha}=\frac{\sigma}{n}, \quad x_{2} \cdot \frac{1}{1-\alpha}=(q+1) \frac{2 N}{N-2},
$$

So that

$$
\begin{aligned}
& x_{1}=\frac{\sigma}{n} \frac{2(q+1) \frac{2}{N-2}}{2(q+1) \frac{N}{N-2}-\frac{\sigma}{n}}, \quad x_{2}=2(q+1)-\frac{\sigma}{n} \frac{2(q+1) \frac{2}{N-2}}{2(q+1) \frac{N}{N-2}-\frac{\sigma}{n}}, \\
& \alpha=\frac{2(q+1) \frac{2}{N-2}}{2(q+1) \frac{N}{N-2}-\frac{\sigma}{n}} .
\end{aligned}
$$

Then the Hölder inequality (1.7) yields

$$
\int_{\Omega} v^{2(q+1)} d x \leq\left(\int_{\Omega} v^{\frac{\sigma}{n}} d x\right)^{\alpha}\left(\int_{\Omega} v^{(q+1) \frac{2 N}{N-2}} d x\right)^{1-\alpha}
$$


We follow the same procedure for $x_{1}^{\prime}, x_{2}^{\prime}$, and $\alpha^{\prime}$, that is, we choose them such that

$$
x_{1}^{\prime}+x_{2}^{\prime}=2(q+1)+\frac{\gamma}{n}, \quad x_{1} \cdot \frac{1}{\alpha^{\prime}}=\frac{\sigma}{n}, \quad x_{2}^{\prime} \cdot \frac{1}{1-\alpha^{\prime}}=(q+1) \frac{2 N}{N-2},
$$

so that

$$
\begin{aligned}
x_{1}^{\prime} & =\frac{\sigma}{n} \frac{2(q+1) \frac{2}{N-2}-\frac{\gamma}{n}}{2(q+1) \frac{N}{N-2}-\frac{\sigma}{n}}, \\
x_{2}^{\prime} & =2(q+1)+\frac{\gamma}{n}-\frac{\sigma}{n} \frac{2(q+1) \frac{2}{N-2}-\frac{\gamma}{n}}{2(q+1) \frac{N}{N-2}-\frac{\sigma}{n}}, \\
\alpha^{\prime} & =\frac{2(q+1) \frac{2}{N-2}-\frac{\gamma}{n}}{2(q+1) \frac{N}{N-2}-\frac{\sigma}{n}},
\end{aligned}
$$

and obtain

$$
\int_{\Omega} v^{2(q+1)+\frac{\gamma}{n}} d x \leq\left(\int_{\Omega} v^{\frac{\sigma}{n}} d x\right)^{\alpha^{\prime}}\left(\int_{\Omega} v^{(q+1) \frac{2 N}{N-2}} d x\right)^{1-\alpha^{\prime}} .
$$

Stressing the Sobolev inequality gives $W_{0}^{1,2} \hookrightarrow L^{\frac{2 N}{N-2}}$ for $N \geq 3$. Consequently, we get

$$
\left\|v^{q+1}\right\|_{L^{\frac{2 N}{N-2}}}^{\frac{2 N}{N-2}(1-\alpha)} \leq c_{1}^{\frac{2 N}{N-2}(1-\alpha)}\left\|\nabla v^{q+1}\right\|_{L^{2}}^{\frac{2 N}{N-2}(1-\alpha)}
$$

and

$$
\left\|v^{q+1}\right\|_{L^{N-2}}^{\frac{2 N}{N-2}\left(1-\alpha^{\prime}\right)} \leq c_{1}^{\frac{2 N}{N-2}\left(1-\alpha^{\prime}\right)}\left\|\nabla v^{q+1}\right\|_{L^{2}}^{\frac{2 N}{N-2}\left(1-\alpha^{\prime}\right)},
$$

where $c_{1}$ is the best embedding constant (see [18]).

A combination of (2.9) and (2.11) leads to

$$
\int_{\Omega} v^{2(q+1)} d x \leq c_{1}^{\frac{2 N(1-\alpha)}{N-2}}\left(\int_{\Omega} v^{\frac{\sigma}{n}} d x\right)^{\alpha}\left(\int_{\Omega}\left|\nabla v^{q+1}\right|^{2} d x\right)^{\frac{N(1-\alpha)}{N-2}} .
$$

An application of the Young inequality yields

$$
\begin{aligned}
\int_{\Omega} v^{2(q+1)} d x \leq & \frac{N \alpha-2}{N-2} c_{1}^{\frac{2 N(1-\alpha)}{N \alpha-2}} \varepsilon_{1}^{-\frac{N(1-\alpha)}{N \alpha-2}}\left(\int_{\Omega} v^{\frac{\sigma}{n}} d x\right)^{\frac{(N-2) \alpha}{N \alpha-2}} \\
& +\frac{N(1-\alpha)}{N-2} \varepsilon_{1} \int_{\Omega}\left|\nabla v^{q+1}\right|^{2} d x
\end{aligned}
$$

where $\varepsilon_{1}$ is a positive constant to be determined later.

A combination of (2.9) and (2.11) also leads to

$$
\begin{aligned}
\int_{\Omega} v^{2(q+1)+\frac{\gamma}{n}} d x \leq & \frac{N \alpha^{\prime}-2}{N-2} c_{1}^{\frac{2 N\left(1-\alpha^{\prime}\right)}{N \alpha^{\prime}-2}} \varepsilon_{2}^{-\frac{N\left(1-\alpha^{\prime}\right)}{N \alpha^{\prime}-2}}\left(\int_{\Omega} v^{\frac{\sigma}{n}} d x\right)^{\frac{(N-2) \alpha^{\prime}}{N \alpha^{\prime}-2}} \\
& +\frac{N\left(1-\alpha^{\prime}\right)}{N-2} \varepsilon_{2} \int_{\Omega}\left|\nabla v^{q+1}\right|^{2} d x
\end{aligned}
$$

where $\varepsilon_{2}$ is a positive constant to be determined later. 
Combining (2.8), (2.14), and (2.15), we obtain

$$
\begin{aligned}
\varphi^{\prime}(t) \leq & -\left[\frac{\sigma(\sigma-1) b_{2}}{n^{2(q+1)}(q+1)}-\frac{q \sigma(\sigma-1) b_{2}}{n^{2(q+1)}} \frac{N(1-\alpha)}{N-2} \varepsilon_{1}-\sigma a_{2} \frac{N\left(1-\alpha^{\prime}\right)}{N-2} \varepsilon_{2}\right] \int_{\Omega}\left|\nabla v^{q+1}\right|^{2} d x \\
& +\sigma a_{1}|\Omega|^{\frac{1}{\sigma}}[\phi(t)]^{\frac{\sigma-1}{\sigma}}+\frac{N \alpha-2}{N-2} c_{1}^{\frac{2 N(1-\alpha)}{N \alpha-2}} \varepsilon_{1}^{-\frac{N(1-\alpha)}{N \alpha-2}} \frac{q \sigma(\sigma-1) b_{2}}{n^{2(q+1)}}[\phi(t)]^{\frac{(N-2) \alpha}{N \alpha-2}} \\
& +\frac{N \alpha^{\prime}-2}{N-2} c_{1}^{\frac{2 N\left(1-\alpha^{\prime}\right)}{N \alpha^{\prime}-2}} \varepsilon_{2}^{-\frac{N\left(1-\alpha^{\prime}\right)}{N \alpha^{\prime}-2}}[\phi(t)]^{\frac{(N-2) \alpha^{\prime}}{N \alpha^{\prime}-2}}
\end{aligned}
$$

By choosing $\varepsilon_{1}$ and $\varepsilon_{2}$ small enough such that

$$
\frac{\sigma(\sigma-1) b_{2}}{n^{2(q+1)}(q+1)}-\frac{q \sigma(\sigma-1) b_{2}}{n^{2(q+1)}} \frac{N(1-\alpha)}{N-2} \varepsilon_{1}-\sigma a_{2} \frac{N\left(1-\alpha^{\prime}\right)}{N-2} \varepsilon_{2} \geq 0
$$

we get the differential inequality

$$
\varphi^{\prime}(t) \leq \sigma a_{1}|\Omega|^{\frac{1}{\sigma}}[\phi(t)]^{\frac{\sigma-1}{\sigma}}+k_{1}[\phi(t)]^{\frac{(N-2) \alpha}{N \alpha-2}}+k_{2}[\phi(t)]^{\frac{(N-2) \alpha^{\prime}}{N \alpha^{\prime}-2}}
$$

with $k_{1}=\frac{N \alpha-2}{N-2} c_{1}^{\frac{2 N(1-\alpha)}{N \alpha-2}} \varepsilon_{1}^{-\frac{N(1-\alpha)}{N \alpha-2}}$ and $k_{2}=\frac{N \alpha^{\prime}-2}{N-2} c_{1}^{\frac{2 N\left(1-\alpha^{\prime}\right)}{N \alpha^{\prime}-2}} \varepsilon_{2}^{-\frac{N\left(1-\alpha^{\prime}\right)}{N \alpha^{\prime}-2}}$.

Inequality (2.18) can be rewritten as

$$
\frac{d \phi}{\sigma a_{1}|\Omega|^{\frac{1}{\sigma}}[\phi(t)]^{\frac{\sigma-1}{\sigma}}+k_{1}[\phi(t)]^{\frac{(N-2) \alpha}{N \alpha-2}}+k_{2}[\phi(t)]^{\frac{(N-2) \alpha^{\prime}}{N \alpha^{\prime}-2}}} \leq d t .
$$

An integration of (2.19) from 0 to $t$ leads to

$$
\int_{\phi(0)}^{\phi(t)} \frac{d \xi}{\sigma a_{1}|\Omega|^{\frac{1}{\sigma}}[\xi]^{\frac{\sigma-1}{\sigma}}+k_{1}[\xi]^{\frac{(N-2) \alpha}{N \alpha-2}}+k_{2}[\xi]^{\frac{(N-2) \alpha^{\prime}}{N \alpha^{\prime}-2}}} \leq t
$$

Taking the limit as $t \longrightarrow t^{*}$, we obtain

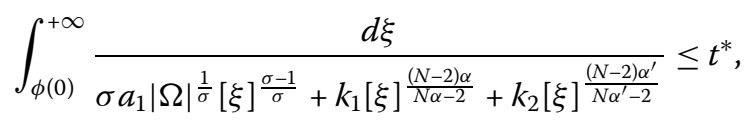

and the proof is complete.

\section{Competing interests}

The authors declare that they have no competing interests.

\section{Authors' contributions}

The authors declare that the study was realized in collaboration with the same responsibility. All authors read and approved the final manuscript.

\section{Acknowledgements}

The authors would like to express their gratitude to the anonymous referees for helpful and very careful reading this paper. The work was supported by the National Natural Science Foundation of China (Grant $\sharp 11471126$ ), Foundation for the Training of the Excellent Young Teachers in Higher Education of Guangdong, China (Grant $\sharp$ Yq2013121), the Project of innovation and strengthen of the University of Guangdong University of Finance (Grant $\sharp 3-7$ ), and Special Funds for the Cultivation of Guangdong College Student's Scientific and Technological Innovation ('Climbing Program' Special Funds). 
References

1. Payne, LE, Philippin, GA, Schaefer, PW: Blow-up phenomena for some nonlinear parabolic problems. Nonlinear Anal. 69, 3495-3502 (2008)

2. Bao, AG, Song, XF: Bounds for the blow up time of the solutions to quasi-linear parabolic problems. Z. Angew. Math Phys. 65, 115-123 (2014)

3. Li, HX, Gao, WJ, Han, YZ: Lower bounds for the blow up time of solutions to a nonlinear parabolic problems. Electron. J. Differ. Equ. 2014, 20 (2014)

4. Liu, DM, Mu, CL, Qiao, X: Lower bounds estimate for the blow up time of a nonlinear nonlocal porous medium equation. Acta Math. Sci. 32, 1206-1212 (2012)

5. Song, JC: Lower bounds for blow-up time in a non-local reaction-diffusion problem. Appl. Math. Lett. 5, 793-796 (2011)

6. Payne, LE, Song, JC: Lower bounds for blow-up time in a nonlinear parabolic problem. J. Math. Anal. Appl. 354, 394-396 (2009)

7. Payne, LE, Schaefer, PW: Lower bounds for blow-up time in parabolic problems under Dirichlet conditions. J. Math. Anal. Appl. 328, 1196-1205 (2007)

8. Payne, LE, Schaefer, PW: Lower bounds for blow-up time in parabolic problems under Neumann conditions. Appl. Anal. 85, 1301-1311 (2006)

9. Payne, LE, Philippin, GA, Schaefer, PW: Bounds for blow-up time in nonlinear parabolic problems. J. Math. Anal. Appl. 338, 438-447 (2008)

10. Payne, LE, Song, JC: Lower bounds for the blow-up time in a temperature dependent Navier-Stokes flow. J. Math. Anal. Appl. 335, 371-376 (2007)

11. Li, YF, Liu, Y, Lin, CH: Blow-up phenomena for some nonlinear parabolic problems under mixed boundary conditions. Nonlinear Anal., Real World Appl. 11, 3815-3823 (2010)

12. Payne, LE, Schaefer, PW: Blow-up in parabolic problems under Robin boundary conditions. Appl. Anal. 87, 699-707 (2008)

13. Ding, J: Global and blow-up solutions for nonlinear parabolic equations with Robin boundary conditions. Comput. Math. Appl. 65(11), 1808-1822 (2013)

14. Enache, C: Blow-up phenomena for a class of quasilinear parabolic problems under Robin boundary condition. Appl. Math. Lett. 24(3), 288-292 (2011)

15. Schaefer, PW: Blow up phenomena in some porous medium problems. Dyn. Syst. Appl. 18, 103-110 (2009)

16. Payne, LE, Schaefer, PW: Bounds for the blow-up time for the heat equation under nonlinear boundary conditions. Proc. R. Soc. Edinb. A 139, 1289-1296 (2009)

17. Payne, LE, Philippin, GA, Vernier Piro, S: Blow-up phenomena for a semilinear heat equation with nonlinear boundary condition, I. Z. Angew. Math. Phys. 61, 999-1007 (2010)

18. Gilbarg, D, Trudinger, NS: Elliptic Partial Differential Equations of Second Order. Springer, Berlin (2001)

\section{Submit your manuscript to a SpringerOpen ${ }^{\circ}$ journal and benefit from:}

- Convenient online submission

Rigorous peer review

- Immediate publication on acceptance

- Open access: articles freely available online

- High visibility within the field

- Retaining the copyright to your article 